\title{
Vibronic coupling models for donor- acceptor aggregates using an effective- mode scheme: Application to mixed Frenkel and charge-transfer excitons in oligothiophene aggregates
}

Cite as: J. Chem. Phys. 150, 244114 (2019); https://doi.org/10.1063/1.5100529

Submitted: 17 April 2019 . Accepted: 05 June 2019. Published Online: 27 June 2019

Wjatscheslaw Popp (D), Matthias Polkehn, Keith H. Hughes, Rocco Martinazzo (D), and Irene Burghardt (iD)

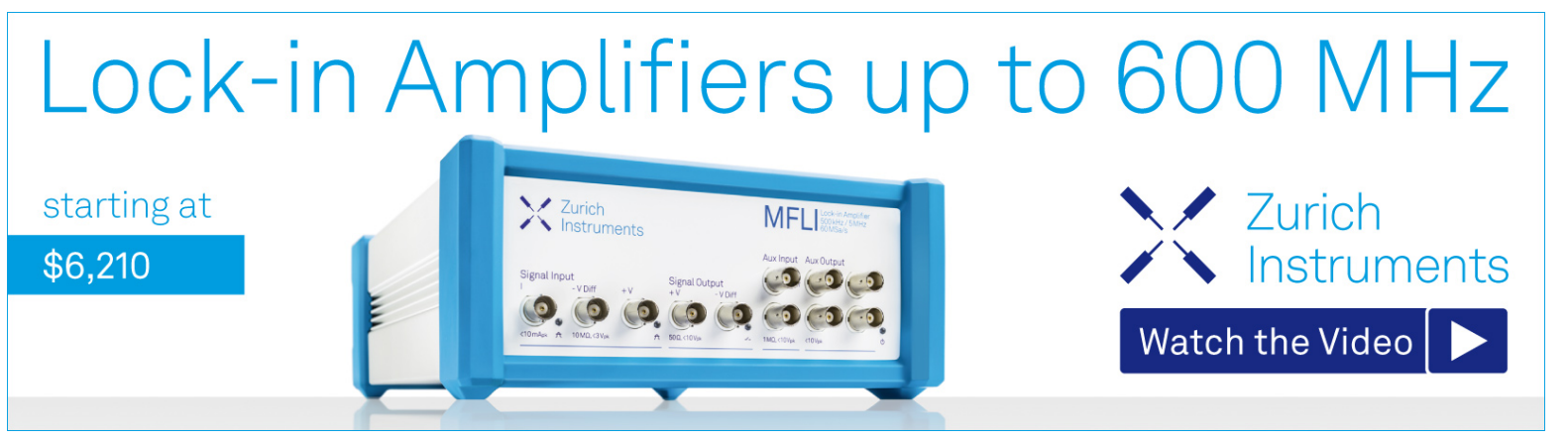




\title{
Vibronic coupling models for donor-acceptor aggregates using an effective-mode scheme: Application to mixed Frenkel and charge-transfer excitons in oligothiophene aggregates
}

Cite as: J. Chem. Phys. 150, 244114 (2019); doi: 10.1063/1.5100529

Submitted: 17 April 2019 • Accepted: 5 June 2019 •

Published Online: 27 June 2019

Wjatscheslaw Popp, (D) Matthias Polkehn, ' Keith H. Hughes, ${ }^{2}$ Rocco Martinazzo, and Irene Burghardt ${ }^{1, a)}$ (D)

\author{
AFFILIATIONS \\ ${ }^{1}$ Institute of Physical and Theoretical Chemistry, Goethe University Frankfurt, Max-von-Laue-Str. 7, 60438 Frankfurt, \\ Germany \\ ${ }^{2}$ School of Natural Sciences, Bangor University, Bangor, Gwynedd LL572UW, United Kingdom \\ ${ }^{3}$ Department of Chemistry, Università degli Studi di Milano, v. Golgi 19, 20133 Milano, Italy
}

a) Electronic mail: burghardt@chemie.uni-frankfurt.de

\begin{abstract}
A reduced-dimensional effective-mode representation is developed in order to efficiently describe excited-state dynamics of multichromophoric donor-acceptor aggregates within a linear vibronic coupling model. Specifically, we consider systems where vibrational modes pertaining to a given molecular fragment couple both to local excitations of Frenkel type and delocalized states of charge transfer exciton type. A hierarchical chain representation is constructed which is suitable to describe correlated fluctuations, leading to a set of correlated spectral densities. An application is shown for a first-principles parameterized model of an oligothiophene H-type aggregate whose properties are modified due to the presence of charge transfer excitons. Within a pentamer model comprising 13 electronic states and 195 normal modes, good convergence of the effective-mode representation of the spectral densities is achieved at the eighth order of the hierarchy with 104 modes, and a qualitatively correct picture is obtained at the sixth order with 78 modes.
\end{abstract}

Published under license by AIP Publishing. https://doi.org/10.1063/1.5100529

\section{INTRODUCTION}

The explicit theoretical treatment of photoinduced quantum dynamics of molecular aggregates is a current issue in many fields of biological, chemical, and materials science. ${ }^{1,2}$ Examples are the photophysics of DNA fragments, ${ }^{3,4}$ light-harvesting systems, ${ }^{5,6}$ and other complexes of biological importance. Another class of relevant systems is conjugated polymer materials, especially in view of their use in organic electronics. ${ }^{7-9}$ In the present study, we focus on the latter class of systems and specifically address the photophysics of regioregular poly-(3-hexylthiophene) (rrP3HT) assemblies, ${ }^{10-15}$ which represent paradigm donor materials in organic photovoltaics.

Over the past few years, it has become increasingly clear that electron-phonon coupling plays a key role in the photophysics of semiconducting polymers, ${ }^{16,17}$ and conventional rate theories turn out insufficient to describe the primary electronic conversion and transport steps. ${ }^{18}$ Hence, a quantum dynamical treatment is in order, comprising a large number of electronic states and vibrational modes. Yet, the explicit quantum dynamical treatment of all vibrational modes and the construction of full-dimensional potential energy surfaces (PESs) are prohibitive 
already for a single polyatomic molecule. For example, a quater-3hexylthiophene oligomer already features about 300 molecular normal modes (NMs). Even with state-of-the-art wavefunction propagation methods like the Multi-Configuration Time-Dependent Hartree (MCTDH) method ${ }^{19-22}$ and its multilayer ML-MCTDH extension, ${ }^{23,24}$ a simulation of three stacked oligomers is a tall order.

To circumvent this problem, it is necessary to (i) simplify the representation of the excited-state PESs and (ii) reduce the highdimensional vibrational space. For large molecular aggregates, the first aspect can often be accomplished using a Linear Vibronic Coupling (LVC) model, ${ }^{25}$ assuming that large-amplitude motions do not play a dominant role. The LVC model represents excited-state PESs in the basis of the ground-state normal mode space, focusing on the shift of the state-dependent equilibrium geometries and neglecting second-order modifications of the PES. The LVC model can also be understood as a generalization of the ubiquitous spin-boson model ${ }^{26}$ and directly connects to system-bath theory formulations in terms of spectral densities (SDs). ${ }^{1,}$

The second issue-i.e., the reduction of the vibrational spacecan be achieved by different approaches. A straightforward strategy is to discard vibrational modes that have a minor influence on the electronic subsystem. A less biased strategy is an effective mode representation that has been developed in the context of the LVC model. ${ }^{27-30}$ In the effective mode representation, an orthogonal transformation is constructed such as to identify a small number of first-layer effective modes (FLEMs) that couple directly to the electronic subsystem, while the complementary set of residual modes is coupled in turn to the first-layer modes. The first-layer modes have been shown to subsume the short-time evolution of the vibronic system. ${ }^{27-30}$ Inclusion of the residual modes permits the successive unraveling of the dynamics as a function of time if a hierarchical, Mori type $^{31}$ chain construction is employed. ${ }^{32-35}$ This also suggests systematic truncation schemes by discarding chain modes beyond a chosen order. From a complementary perspective, this scheme can be viewed as an unraveling of non-Markovian system-environment dynamics in terms of a hierarchical chain representation of the memory kernel. ${ }^{36-41}$ In the frequency domain, truncated chain representations yield a series of SD approximants which converge to the exact SD. . $^{38,41,42}$

Effective-mode methods have been employed either in cases where the subsystem consists of a single electronic operator or vibrational mode ${ }^{36-40}$ or in situations where several subsystem operators are present. ${ }^{27,34,42}$ In the latter category, the focus was on conical intersection topologies where vibronically active modes can couple both diagonally and off-diagonally to the electronic subsystem in a diabatic representation. ${ }^{27-30,42}$ In the present work, we introduce a related construction scheme which is suitable to describe photoexcitations in molecular aggregates. ${ }^{1}$ Specifically, we consider multichromophoric donor-acceptor aggregates where vibrational modes pertaining to a given molecular fragment couple both to local excitations (typically of Frenkel type) and delocalized states (typically of charge transfer type). This situation gives rise to correlated fluctuations that are captured by correlated spectral densities and their effective-mode representations. ${ }^{42}$

As a representative model system, we choose a small $\mathrm{H}$-aggregate ${ }^{14}$ composed of quaterthiophene oligomers, i.e., (OT4) with $n=2, \ldots 5$, representative of $\operatorname{rrP} 3 \mathrm{HT}$. This type of aggregate can be described as an $\mathrm{H}$-aggregate of Frenkel excitonic (XT) states with a strong admixture of charge transfer excitons (CTXs). ${ }^{14,43}$ The pronounced participation of CTX species has been reported in various time-independent and time-resolved spectroscopic studies. ${ }^{16,44,45}$ The aggregate model Hamiltonian is parameterized based upon Time-Dependent Density Functional Theory (TDDFT) electronic structure calculations for an (OT4) $)_{2}$ species, ${ }^{46}$ and quantum dynamical calculations are subsequently performed for larger $(\mathrm{OT} 4)_{n}$ systems up to $n=5 .^{47}$ In the present paper, the performance of the effective mode transformation will be evaluated by comparing the resulting effective-mode SDs for the dimer $(\mathrm{OT} 4)_{2}$ and pentamer (OT4) 5 with the SDs obtained for the full normal mode space. In a companion study, we report on high-dimensional quantum dynamical simulations for an $(\mathrm{OT} 4)_{5}$ pentamer system using the ML-MCTDH method.

The remainder of the paper is organized as follows. Section II introduces the multisite LVC model employed in this work, and Sec. III details the effective-mode approach. The parameterization of the (OT4 $)_{5}$ system resulting from electronic structure calculations and the performance of the effective mode representation are described in Sec. IV. Finally, Sec. V summarizes our findings. The supplementary material presents additional information on the electronic structure calculations and effective mode analysis.

\section{VIBRONIC LATTICE MODEL FOR MULTICHROMOPHORIC SYSTEMS}

Following Refs. 17, 46 and 48, we employ a first-principles parameterized vibronic lattice Hamiltonian to describe a multichromophoric system exhibiting a pronounced mixture of XT and CTX states. We specifically consider a model system consisting of five quaterthiophene molecules stacked on top of each other, i.e., an $(\mathrm{OT} 4)_{5}$ aggregate, as illustrated in Fig. 1. While the model is taken to be restricted to homo-aggregates with identical monomer species, a generalization to hetero-aggregates is straightforward.

Figure 1 shows the molecular geometry of the system as well as a schematic sketch of the electronic and vibronic couplings, shown as connectivities between electronic states and vibrational modes. Each OT4 fragment, denoted monomer $M_{n}$ in the following, is associated with its own subset of normal modes $\boldsymbol{q}_{M_{n}}$, which we will refer to as local normal modes (local NMs). Furthermore, the associated state-specific sets of vibronic couplings are obtained from electronic structure calculations, and coordinate-independent electronic couplings are obtained via a quasidiabatization scheme. ${ }^{49}$ Additional details on the electronic structure aspects are provided in Sec. IV.

The corresponding model Hamiltonian is set up in a single excitation subspace in an electron-hole $(e-h)$ representation, using basis states $|v \mu\rangle$ with the electron located at site $v_{e}=v$ and the hole located at site $\mu_{h}=\mu$. Localized $e$ - $h$ pairs where the electron and hole are located on the same molecular fragment correspond to a Frenkel excitonic (XT) configuration, $\left|\mathrm{XT}_{n}\right\rangle=|v=n, \mu=n\rangle$ (with $n=1, \ldots, N$ for an $N$-site system). Conversely, if an $e$ - $h$ pair is spatially separated, the configuration represents a charge transfer exciton (CTX) state, i.e., $\left|\mathrm{CTX}_{n, n^{\prime}}\right\rangle=\left|v=n, \mu=n^{\prime}\right\rangle$, where $n \neq n^{\prime}$. In the present study, we make the approximation that the electron and hole are always located on neighboring fragments, i.e., only $\left|\mathrm{CTX}_{n, n^{\prime}}\right\rangle$ states with $n^{\prime}=n \pm 1$ will be considered. Hence, nearest-neighbor 
a)
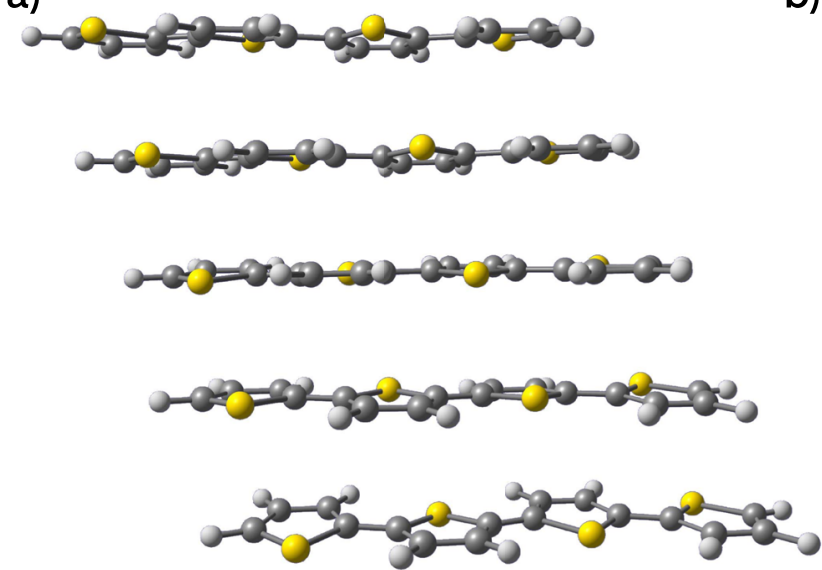

b)

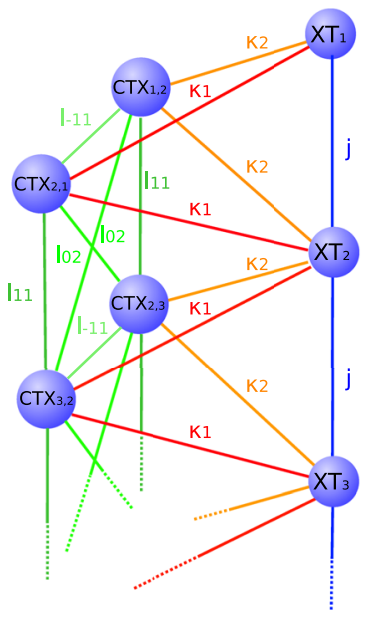

c)

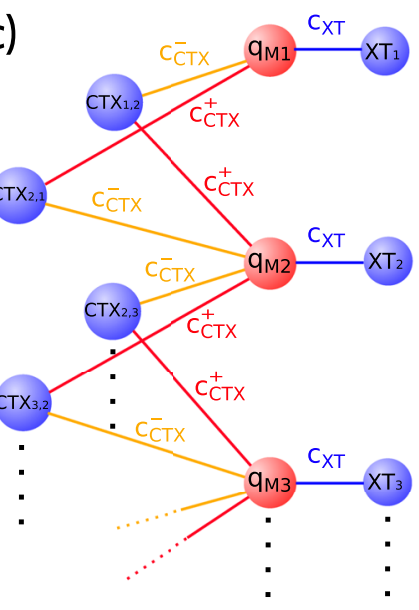

FIG. 1. (a) Molecular representation of the stacked (OT4) 5 pentamer aggregate at the center of the present studies. (b) Schematic representation of the electronic couplings in the aggregate system, involving XT and CTX states (blue circles). Various types of electronic couplings appearing in Eqs. (4)-(6) are marked along the edges connecting two nodes describing electronic states. (c) Schematic representation of the vibronic couplings of Eqs. (14) and (15) where the monomer-specific vibrations are shown as additional nodes (red circles), the electronic states appear as in panel b (blue circles), and the relevant local ( $\left.c_{\mathrm{XT}}\right)$ and nonlocal ( $c_{\mathrm{CTX}}^{ \pm}$) vibronic couplings are marked along the edges connecting two nodes of vibrational (blue) and electronic (red) types.

electron transfer pathways, $\left|\mathrm{XT}_{n}\right\rangle \rightarrow\left|\mathrm{CTX}_{n \pm 1, n}\right\rangle$, are distinguished from the corresponding hole transfer pathways, $\left|\mathrm{XT}_{n}\right\rangle \rightarrow\left|\mathrm{CTX}_{n, n \pm 1}\right\rangle$. The nearest-neighbor approximation is justified by our previous study ${ }^{46}$ which showed that longer-range charge separation within an OT aggregate does not play a prominent role. In the present work, up to five stacked OT4 monomers are included, resulting in 13 electronic states, i.e., five XT states and eight CTX states.

The overall Hamiltonian $\widehat{H}$ can be split into three terms

$$
\widehat{H}=\widehat{H}^{\mathrm{el}}+\widehat{H}^{\mathrm{ph}}+\widehat{H}^{\mathrm{e}-\mathrm{ph}},
$$

where the first term $\left(\widehat{H}^{\mathrm{el}}\right)$ corresponds to the purely electronic part of the Hamiltonian including the electronic couplings, while the second term $\left(\widehat{H}^{\mathrm{ph}}\right)$ represents the zeroth-order vibrational (phonon) Hamiltonian. The third term $\left(\widehat{H}^{\mathrm{e}-\mathrm{ph}}\right)$ subsumes all vibronic interactions. These terms will be successively addressed in the following.

\section{A. Electronic Hamiltonian}

The electronic part of the Hamiltonian is in turn split into four contributions

$$
\widehat{H}^{\text {el }}=\widehat{H}^{\text {on-site }}+\widehat{H}_{\mathrm{XT}}^{\text {coup }}+\widehat{H}_{\mathrm{XT}, \mathrm{CTX}}^{\text {coup }}+\widehat{H}_{\mathrm{CTX}}^{\text {coup }} .
$$

The first contribution, i.e., the on-site Hamiltonian $\widehat{H}^{\text {on-site }}$, contains the static energy shifts of XT and CTX states within an aggregate composed of $N$ monomers,

$$
\widehat{H}^{\text {on-site }}=\sum_{n=1}^{N}\left(\epsilon_{\mathrm{XT}}\left|\mathrm{XT}_{n}\right\rangle\left\langle\mathrm{XT}_{n}\left|+\epsilon_{\mathrm{CTX}}\right| \mathrm{CTX}_{n, n \pm 1}\right\rangle\left\langle\mathrm{CTX}_{n, n \pm 1}\right|\right),
$$

with $1 \leq n \pm 1 \leq N$. Here, all Frenkel states have the same on-site energy $\epsilon_{\mathrm{XT}}$ and all nearest-neighbor charge-separated states have the same on-site energy $\epsilon_{\mathrm{CTX}}$.
The remaining terms in Eq. (2) describe several types of electronic couplings, including the excitonic (Frenkel type) coupling between two neighboring XT states,

$$
\widehat{H}_{\mathrm{XT}}^{\text {coup }}=j \sum_{n=1}^{N}\left(\left|\mathrm{XT}_{n}\right\rangle\left\langle\mathrm{XT}_{n+1}\right|+\text { h.c. }\right),
$$

and the coupling between XT and CTX states,

$$
\widehat{H}_{\mathrm{XT}, \mathrm{CTX}}^{\text {coup }}=\sum_{n=1}^{N}\left(\kappa_{1}\left|\mathrm{XT}_{n}\right\rangle\left\langle\mathrm{CTX}_{n \pm 1, n}\left|+\kappa_{2}\right| \mathrm{XT}_{n}\right\rangle\left\langle\mathrm{CTX}_{n, n \pm 1}\right|+\text { h.c. }\right) \text {, }
$$

again with $1 \leq n \pm 1 \leq N$. That is, XT states are coupled to CTX states with either the electron or the hole displaced to a neighboring site such that $\kappa_{1}$ is associated with electron transfer and $\kappa_{2}$ is associated with hole transfer. Furthermore, the Hamiltonian Eq. (2) includes the coupling between CTX states,

$$
\begin{aligned}
\widehat{H}_{\mathrm{CTX}}^{\text {coup }}= & \sum_{n, m, m^{\prime}=1}^{N} l_{\Delta m \Delta m^{\prime}}\left(\left|\mathrm{CTX}_{n+1, n}\right\rangle\left\langle\mathrm{CTX}_{m, m^{\prime}}\right|\right. \\
& \left.+\left|\mathrm{CTX}_{n, n+1}\right\rangle\left\langle\mathrm{CTX}_{m^{\prime}, m}\right|+\text { h.c. }\right),
\end{aligned}
$$

where $\left|m-m^{\prime}\right|=1$ and the two indices of the couplings $l_{\Delta m \Delta m^{\prime}}$ indicate the shift in electron and hole positions, i.e., $\Delta_{m}=m-(n+1)$ and $\Delta_{m^{\prime}}=m^{\prime}-n$. We specifically include the couplings with $\left(\Delta_{m}, \Delta_{m^{\prime}}\right)=(1,1),(0,2),(-1,1)$; see Table I and also Fig. S4 of the supplementary material.

The electronic Hamiltonian of Eqs. (2)-(6) can be rewritten in the matrix form, highlighting the coupling between the different excitonic and charge transfer states. Here, we specifically show the pentamer Hamiltonian, $N=5$, which is at the center of our study, 


$$
\boldsymbol{H}^{\mathrm{el}}=\left(\begin{array}{ccccccccccccc}
\epsilon_{\mathrm{XT}} & j & & & & \kappa_{1} & & & & \kappa_{2} & & & \\
j & \epsilon_{\mathrm{XT}} & j & & & \kappa_{2} & \kappa_{1} & & & \kappa_{1} & \kappa_{2} & & \\
& j & \epsilon_{\mathrm{XT}} & j & & & \kappa_{2} & \kappa_{1} & & & \kappa_{1} & \kappa_{2} & \\
& & j & \epsilon_{\mathrm{XT}} & j & & & \kappa_{2} & \kappa_{1} & & & \kappa_{1} & \kappa_{2} \\
& & & j & \epsilon_{\mathrm{XT}} & & & & \kappa_{2} & & & & \kappa_{1} \\
\kappa_{1} & \kappa_{2} & & & & \epsilon_{\mathrm{CTX}} & l_{11} & & & l_{-11} & l_{02} & & \\
& \kappa_{1} & \kappa_{2} & & & l_{11} & \epsilon_{\mathrm{CTX}} & l_{11} & & & l_{-11} & l_{02} & \\
& & \kappa_{1} & \kappa_{2} & & & l_{11} & \epsilon_{\mathrm{CTX}} & l_{11} & & & l_{-11} & l_{02} \\
& & & \kappa_{1} & \kappa_{2} & & & l_{11} & \epsilon_{\mathrm{CTX}} & & & & l_{-11} \\
\kappa_{2} & \kappa_{1} & & & & l_{-11} & & & & \epsilon_{\mathrm{CTX}} & l_{11} & & \\
& \kappa_{2} & \kappa_{1} & & & l_{02} & l_{-11} & & & l_{11} & \epsilon_{\mathrm{CTX}} & l_{11} & \\
& & \kappa_{2} & \kappa_{1} & & & l_{02} & l_{-11} & & & l_{11} & \epsilon_{\mathrm{CTX}} & l_{11} \\
& & & \kappa_{2} & \kappa_{1} & & & l_{02} & l_{-11} & & & l_{11} & \epsilon_{\mathrm{CTX}}
\end{array}\right) .
$$

In Eq. (7), the following sequence of basis states was chosen: $\left\{\left|\mathrm{XT}_{1}\right\rangle \ldots,\left|\mathrm{XT}_{5}\right\rangle,\left|\mathrm{CTX}_{1,2}\right\rangle \ldots\left|\mathrm{CTX}_{4,5}\right\rangle,\left|\mathrm{CTX}_{2,1}\right\rangle \ldots\left|\mathrm{CTX}_{5,4}\right\rangle\right\}$.

As further detailed in Sec. IV, all parameters appearing in Eq. (7), i.e., the on-site energies and diabatic electronic couplings employed for our (OT4) ${ }_{n}$ models, are based on TDDFT calculations.

\section{B. Vibronic interactions}

The vibrational and vibronic coupling parts of the Hamiltonian are constructed in mass and frequency weighted coordinates such that the zeroth-order vibrational (or phonon) part of the Hamiltonian Eq. (1) reads

$$
\widehat{H}^{\mathrm{ph}}=\frac{1}{2}\left(\widehat{\mathbf{p}}^{T} \boldsymbol{\omega} \widehat{\mathbf{p}}+\widehat{\mathbf{q}}^{T} \boldsymbol{\omega} \widehat{\mathbf{q}}\right),
$$

where $\boldsymbol{\omega}$ is a diagonal frequency matrix in the local NM representation, $(\boldsymbol{\omega})_{i j}=\omega_{i} \delta_{i j}$, and the $\widehat{\mathbf{q}}$ and $\widehat{\mathbf{p}}$ vectors refer to the total normal mode space which is spanned by $N$ monomeric (local) NM subspaces,

$$
\widehat{\mathbf{q}}^{T}=\left(\widehat{\mathbf{q}}_{M_{1}}^{T}, \widehat{\mathbf{q}}_{M_{2}}^{T}, \cdots, \widehat{\mathbf{q}}_{M_{N-1}}^{T}, \widehat{\mathbf{q}}_{M_{N}}^{T}\right),
$$

where the index $M_{N}$ denotes the $M_{N}$ th monomer and $\widehat{\mathbf{q}}_{M}^{T}$ $=\left(\widehat{q}_{M_{n}, 1}, \ldots, \widehat{q}_{M_{n}, N_{M}}\right)$, with $N_{M}$ the number of phonon modes for a

TABLE I. On-site energies and diabatic couplings obtained from TDDFT calculations $(\omega \mathrm{B} 97 \mathrm{XD} / / \mathrm{SVP})$ for an $(\mathrm{OT} 4)_{2}$ dimer. All parameters are given in $\mathrm{eV}$.

\begin{tabular}{lccccccc}
\hline \multicolumn{10}{l}{$\begin{array}{l}\text { On-site } \\
\text { energies }(\mathrm{eV})\end{array}$} & \multicolumn{7}{c}{$\begin{array}{c}\text { Diabatic } \\
\text { couplings }(\mathrm{eV})\end{array}$} \\
\hline$\epsilon_{\mathrm{XT}}$ & $\epsilon_{\mathrm{CT}}$ & $\kappa_{1}$ & $\kappa_{2}$ & $j$ & $l_{11}$ & $l_{02}$ & $l_{-11}$ \\
\hline 0.00 & 0.41 & 0.21 & -0.06 & 0.09 & -0.12 & -0.06 & 0.06 \\
\hline \hline
\end{tabular}

given fragment (which is equal for all fragments in the present case). Hence, the dimensionality of the $\widehat{\mathbf{q}}$ vector corresponds to the total number of phonon (vibrational) modes, $N_{\mathrm{ph}}=N N_{M}$.

Vibronic interactions are defined within the shifted harmonic oscillator approximation of the LVC model for $N_{s}$ electronic states,

$$
\widehat{H}^{\mathrm{e}-\mathrm{ph}}=\sum_{s=1}^{N_{s}} \boldsymbol{C}_{s}^{T} \widehat{\mathbf{q}}|s\rangle\langle s|
$$

where $s$ runs over the manifolds of XT and CTX states. The statespecific vibronic couplings $\left\{C_{s, j}\right\}, j=1, \ldots, N_{\mathrm{ph}}$, are calculated from Franck-Condon (FC) gradients of every state of interest. While all modes are taken to be local in our model-i.e., localized on a given molecular fragment-the vibronic couplings may be nonlocal, depending on the nature of the excited state. That is, we refer to local vibronic interactions in the case of Frenkel XT states, while nonlocal vibronic interactions arise in the case of charge-transfer excitons, i.e., CTX states. In the latter case, modes $\widehat{\mathbf{q}}_{M_{n}}$ belonging to the $n$th fragment also couple to the $(n+1)$-th and $(n-1)$-th fragment due to the delocalized nature of the CTX states. Hence, we split the electron-phonon coupling term into two contributions

$$
\widehat{H}^{\mathrm{e}-\mathrm{ph}}=\widehat{H}_{\text {local }}^{\mathrm{e}-\mathrm{ph}}+\widehat{H}_{\text {non-local }}^{\mathrm{e}-\mathrm{ph}}
$$

with the local vibronic couplings

$$
\widehat{H}_{\text {local }}^{\text {e-ph }}=\sum_{n=1}^{N} C_{\mathrm{XT}, n}^{T} \widehat{\mathbf{q}}\left|\mathrm{XT}_{n}\right\rangle\left\langle\mathrm{XT}_{n}\right|
$$

and the nonlocal vibronic couplings

$$
\widehat{H}_{\text {non-local }}^{\text {e-ph }}=\sum_{\substack{n=1 \\ n^{\prime}=1 \\ n^{\prime} \neq n}}^{N} \boldsymbol{C}_{\mathrm{CTX}, n n^{\prime}}^{T} \widehat{\mathbf{q}}\left|\mathrm{CTX}_{n, n^{\prime}}\right\rangle\left\langle\mathrm{CTX}_{n, n^{\prime}}\right| .
$$

The respective vibronic coupling vectors are given as

$$
\boldsymbol{C}_{\mathrm{XT}, n}^{T}=\left(\mathbf{0}^{(n-1)}, \boldsymbol{c}_{\mathrm{XT}}^{T}, \mathbf{0}^{(N-n)}\right),
$$


where the modes of the $n$th monomer couple to the $n$th Frenkel states with vibronic couplings $\boldsymbol{c}_{\mathrm{XT}}^{T}=\left(c_{\mathrm{XT}, 1}, \ldots, c_{\mathrm{XT}, N_{M}}\right)$ and

$$
\boldsymbol{C}_{\mathrm{CTX}, n n^{\prime}}^{T}=\left(\mathbf{0}^{(n-1)}, \boldsymbol{c}_{\mathrm{CTX}}^{( \pm) T}, \boldsymbol{c}_{\mathrm{CTX}}^{(\mp) T}, \mathbf{0}^{(N-n-1)}\right),
$$

where the modes of the $n$th and $(n+1)$-th monomer states couple to the negatively (positively) charged $n$th monomer and the positively (negatively) charged $(n+1)$-th monomer, with different vibronic couplings $\boldsymbol{c}_{\mathrm{CTX}}^{(-)}$and $\boldsymbol{c}_{\mathrm{CTX}}^{(+)}$. Each of the full vibronic coupling vectors $\boldsymbol{C}$ has $N_{\text {ph }}=N N_{M}$ entries, while each subvector $\boldsymbol{c}$ has $N_{M}$ entries. Correspondingly, the $\mathbf{0}^{(m)}$ vectors have length $m N_{M}$. In practice, the $\left(n, n^{\prime}\right)$ combinations are limited to nearest neighbor CTX states with $n^{\prime}=n \pm 1$ as explained above.

\section{Correlated spectral densities}

If the frequency distribution of the vibrational modes is dense, it is natural to characterize the influence of the vibrations on the electronic subsystem in terms of a spectral density or its discretized representation. ${ }^{1,26}$ Due to the nonlocal part of the electron-phonon coupling, the electronic subsystem in the aggregate system under study experiences correlated fluctuations, ${ }^{42}$ which are described by a set of spectral densities resulting from the vibronic coupling Hamiltonian Eq. (10),

$$
J_{s s^{\prime}}(\omega)=\frac{\pi}{2} \sum_{j=1}^{N_{\mathrm{ph}}} C_{s, j} C_{s^{\prime}, j} \delta\left(\omega-\omega_{j}\right),
$$

which define a $N_{s} \times N_{s}$ dimensional spectral density matrix $\boldsymbol{J}$. From the structure of the Hamiltonian, particular elements of $\boldsymbol{J}$ are zero by construction-e.g., in our model the Frenkel-type $\left|\mathrm{XT}_{n}\right\rangle$ states are uncorrelated as long as all vibrational modes are assumed to be of local type.

In the analysis reported below, we will employ Eq. (16) in conjunction with the convolution with a Lorentzian function, generating a series of continuous spectral densities.

\section{EFFECTIVE MODE APPROACH}

For the study of extended systems comprising various molecular fragments, the large number of degrees of freedom is the computational bottleneck of the dynamical simulations. The approach of choice is to reduce the dimension of the vibrational subspace. To this end, we employ the above-mentioned effective mode approach, ${ }^{27,28,34,42}$ which relies on an orthogonal transformation in the vibrational subspace, as a result of which only few collective modes couple to the electronic subsystem. These will be termed firstlayer effective modes (FLEMs) in the following. In molecular aggregates with $N$ monomer units $\left(M_{1}-M_{2}-\cdots-M_{N}\right)$, there are $N_{\mathrm{XT}}$ $=N$ Frenkel excitonic states and $N_{\text {CTX }}=(2 N-2)$ CTX states-i.e., in total, $N_{s}=3 N-2$ electronic states-which leads to the definition of $N_{s}$ FLEMs. In the following, we first consider the construction of the latter and then turn to the full orthogonal coordinate transformation that also includes a set of residual modes.

\section{A. Construction of FLEM space}

The FLEMs are constructed to subsume the state-specific vibronic couplings of Eq. (10) into a single collective coordinate per electronic state,

$$
\widehat{Q}_{s}=\frac{1}{\bar{c}^{s}} C_{s}^{T} \widehat{\mathbf{q}},
$$

where $\bar{c}^{s}$ is a normalization constant. Hence, by construction, the electron-phonon coupling is cast in the form

$$
\widehat{H}^{\mathrm{e}-\mathrm{ph}}=\sum_{s}^{N_{s}} \bar{c}^{s} \widehat{\mathrm{Q}}_{s}|s\rangle\langle s| .
$$

Specifically, $N_{\mathrm{XT}}=N$ effective modes are constructed to capture the vibronic coupling with the Frenkel XT states

$$
\widehat{Q}_{\mathrm{XT}, n}=\frac{1}{\overline{\bar{c}}_{\mathrm{XT}, n}} \boldsymbol{C}_{\mathrm{XT}, n}^{T} \widehat{\mathbf{q}},
$$

where $n=1, \ldots, N$. Furthermore, $N_{\text {CTX }}=2 N-2$ effective modes are constructed to account for the vibronic coupling with the CTX states

$$
\widehat{Q}_{\mathrm{CTX}, n n^{\prime}}=\frac{1}{\bar{c}_{\mathrm{CTX}, n n^{\prime}}} C_{\mathrm{CTX}, n n^{\prime}}^{T} \widehat{\mathbf{q}},
$$

where $n=1, \ldots, N$ and $n^{\prime}=n \pm 1$. As a result, the local and nonlocal parts of the electron-phonon coupling Hamiltonian, $\widehat{H}^{\mathrm{e}-\mathrm{ph}}=\widehat{H}_{\text {local }}^{\mathrm{e} \text {-ph }}+\widehat{H}_{\text {non-local }}^{\mathrm{e} \text {-ph }}$ of Eq. (11), are recast as follows in terms of $3 N-2$ modes:

$$
\widehat{H}_{\text {local }}^{\text {e-ph }}=\sum_{n}^{N} \bar{c}_{\mathrm{XT}, n} \widehat{Q}_{\mathrm{XT}, n}\left|\mathrm{XT}_{n}\right\rangle\left\langle\mathrm{XT}_{n}\right|
$$

and

$$
\widehat{H}_{\text {non-local }}^{\mathrm{e}-\mathrm{ph}}=\sum_{n}^{N} \bar{c}_{\mathrm{CTX}, n n \pm 1} \widehat{\mathrm{Q}}_{\mathrm{CTX}, n n \pm 1}\left|\mathrm{CTX}_{n, n \pm 1}\right\rangle\left\langle\mathrm{CTX}_{n, n \pm 1}\right| .
$$

While the modes $\left\{\widehat{Q}_{\mathrm{XT}, n}\right\}$ are orthogonal to each other, they are not orthogonal to the $\left\{\widehat{Q}_{\mathrm{CTX}, n n^{\prime}}\right\}$ modes. Hence, in a next step, the primary FLEM modes $\widehat{\mathbf{Q}}$ will be orthogonalized. Furthermore, the remaining modes will be included, which couple to the FLEMs and therefore exert an indirect influence on the electronic subsystem.

\section{B. Full coordinate transformation}

The starting point of the full coordinate transformation relating the original set of normal modes and the new set of effective modes is the definition of the orthogonalized FLEM modes which determine the first rows of an orthogonal transformation matrix $\mathbf{T}$,

$$
\widehat{\mathbf{Q}}=\mathbf{T} \widehat{\mathbf{q}}
$$

As a consequence of the transformation Eq. (23) and noting that the transformation can alternatively be formulated such as to comprise the momentum vector,

$$
(\widehat{\mathbf{Q}} \pm i \widehat{\mathbf{P}})=\mathbf{T}(\widehat{\mathbf{q}} \pm \imath \widehat{\mathbf{p}}),
$$

the phonon part of the Hamiltonian, $\widehat{H}^{\text {ph }}$ of Eq. (8), takes the form

$$
\widehat{H}^{\mathrm{ph}}=\frac{1}{2}\left(\widehat{\mathbf{P}}^{T} \Omega \widehat{\mathbf{P}}+\widehat{\mathbf{Q}}^{T} \Omega \widehat{\mathbf{Q}}\right)
$$

with

$$
\boldsymbol{\omega}=\mathbf{T}^{T} \Omega \mathbf{T} .
$$


The diagonal frequency matrix $\boldsymbol{\omega}$ in the local NM space is thus transformed to a nondiagonal form in the effective-mode representation. The diagonal entries of $\boldsymbol{\Omega}$ are the effective mode frequencies, while the off diagonal elements (in the following denoted as $d_{i j}$ ) are intermode couplings whose explicit form is as follows: ${ }^{28,34}$

$$
\Omega_{i}=\sum_{j} \omega_{j} T_{j i}^{2}, \quad d_{i j}=\sum_{k} \omega_{k} T_{k i} T_{k j} .
$$

Up to here, no conditions have been imposed on the matrix $\mathbf{T}$. The most important constraint is to ensure orthonormality of the effective modes so that the columns of $\mathbf{T}$ have to be orthonormal as well. The second constraint results from the definition of the FLEMs according to Eqs. (19) and (20). The first $N_{s}$ columns of the initial matrix $\mathbf{T}$ are thus constructed by the normalized-but only partially orthogonal-vibronic coupling vectors of Eqs. (19) and (20) which span the $N_{s}$-dimensional FLEM space. The remaining columns are initially filled with random numbers and will be orthogonalized in the next step.

The first $N_{\mathrm{XT}}=N$ columns are orthogonal by construction; hence, the remaining $N\left(N_{M}-1\right)$ columns are iteratively orthogonalized with respect to the first $N$ columns,

$$
\begin{aligned}
\mathbf{T}_{: k}^{\text {final }} & =\mathbf{T}_{: k}-\sum_{l=1}^{k-1} \frac{\mathbf{T}_{: k}^{T} \mathbf{T}_{: l}}{\left|\mathbf{T}_{: l}\right|^{2}} \mathbf{T}_{: l} \\
& =\mathbf{T}_{: k}-\sum_{l=1}^{k-1} \Lambda_{l, k} \mathbf{T}_{: l},
\end{aligned}
$$

with $k=N_{X T}+1, \ldots, N N_{M}$. The matrix $\boldsymbol{\Lambda}$ in Eq. (28) contains the Gram-Schmidt factors for the columns to be orthonormalized. This results in the general effective mode transformation matrix by analogy with the construction scheme described in Refs. 28, 33, and 34 .

\section{Transformations in the residual mode space}

As detailed in Refs. 28, 30, 33, and 42, there are several possibilities to reshape the effective mode frequency matrix by additional transformations,

$$
\Omega^{\prime}=Z^{T} \Omega Z \text {. }
$$

One of the possibilities is to band-diagonalize the frequency matrix by sequential Givens rotations, which results in a hierarchical, Moritype $^{31}$ effective mode chain. The effective mode chain can now be truncated at a threshold $K=k N_{s}$, i.e., a multiple of the number of FLEMs. Figure 2 shows a matrix $\boldsymbol{\Omega}^{\prime}$ of this type for $N_{s}=3$ and $K=2 N_{s}=6$, i.e., for two layers of effective modes.

Truncation of the frequency matrix $\boldsymbol{\Omega}^{\prime}$ yields a reduceddimensional $k$ th-order matrix $\boldsymbol{\Omega}^{(k)}$ of dimension $K \times K$. In the present work, we use this type of truncation scheme, which leads to reduction in dimensionality for the dynamical problem. In applications to multichromophoric systems, this scheme turns out to suffice for generating an effectively irreversible dynamics due to efficient decoherence in systems exhibiting vibronic coupling in the presence of multiple electronic subsystem operators. Alternatively, Markovian closure procedures have been suggested.

According to Eq. (25), the off diagonal entries for $\boldsymbol{\Omega}^{\prime}$ or $\boldsymbol{\Omega}^{(k)}$ give rise to coordinate and momentum couplings in the phonon

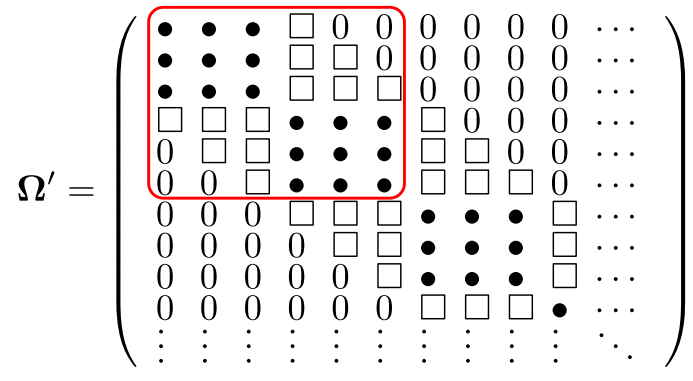

FIG. 2. Schematic representation of a transformed $\boldsymbol{\Omega}^{\prime}$ of Eq. (29) in a banddiagonal form for $N_{s}=3$ and $K=2 N_{s}=6$, i.e., for two layers of effective modes, as indicated by a red frame. Bullets represent the effective mode frequencies and intralayer effective mode couplings on the diagonal, and squares indicate couplings between effective modes of different layers.

part of the Hamiltonian, $\left(\widehat{H}_{\mathrm{ph}}\right)_{i j}=(1 / 2) d_{i j}^{\prime}\left(\widehat{Q}_{i} \widehat{Q}_{j}+\widehat{P}_{i} \widehat{P}_{j}\right)$. In previous applications, these bilinear momentum couplings were found to cause numerical convergence issues and led to increased propagation times. Therefore, an alternative representation may be preferable once the Mori chain representation has been truncated. Following the procedure of Ref. 50, we rediagonalize the truncated chain representation. The final frequency matrix is therefore diagonal,

$$
\boldsymbol{\omega}^{(k)}=\mathbf{U}^{T} \mathbf{\Omega}^{(k)} \mathbf{U},
$$

similar to the original form [Eq. (26)] but in a reduced dimensionality. The representation of Eq. (30) corresponds to the normal mode representation of the truncated chain. In contrast to the local NM representation that we used as a starting point, the truncated chain representation will be seen to yield global normal modes (global NMs) as eigenvectors of $\boldsymbol{\Omega}^{(k)}$. In the following, these global NMs resulting from the $k$ th order truncated representation will be denoted as $\widehat{\mathbf{q}}^{(k)}$.

In this final truncated representation, the electron-phonon coupling Hamiltonian reads as follows:

$$
\widehat{H}_{\text {local }}^{\text {e-ph }}=\sum_{n=1}^{N} \boldsymbol{C}_{\mathrm{XT}, n}^{(k)} \widehat{\mathbf{q}}^{(k)}\left|\mathrm{XT}_{n}\right\rangle\left\langle\mathrm{XT}_{n}\right|
$$

and

$$
\widehat{H}_{\text {non-local }}^{\text {e-ph }}=\sum_{n=1}^{N} \sum_{\substack{n^{\prime}=1 \\ n^{\prime} \neq n}}^{N} \boldsymbol{C}_{\mathrm{CTX}, n n^{\prime}}^{(k) T} \widehat{\mathbf{q}}^{(k)}\left|\mathrm{CTX}_{n, n^{\prime}}\right\rangle\left\langle\mathrm{CTX}_{n, n^{\prime}}\right|,
$$

where

$$
\widehat{\mathbf{q}}^{(k) T}=\left(q_{1}^{(k)}, q_{2}^{(k)}, \ldots, q_{K}^{(k)}\right) .
$$

This is the representation which is most convenient for the numerical implementation.

Equations (31) and (32) are entirely analogous in formal appearance to Eqs. (12) and (13), but the phonon space has been reduced from $N_{\mathrm{ph}}$ to $K=k N_{s}$ modes. The procedure is perhaps best appreciated when the bath comprises an infinitely dense set of modes and the vibronic couplings of Eqs. (12) and (13) become integrals over such modes. Equations (31) and (32) then represent 
quadrature formulas for such an integral, using quadrature pointshere, effective modes-that reproduce the correct dynamical behavior in time.

\section{Spectral density approximants}

A reduced discretized representation of the spectral densities Eq. (16) is naturally obtained from the above truncated chain representation,

$$
J_{s s^{\prime}}^{(k)}(\omega)=\frac{\pi}{2} \sum_{j=1}^{K} C_{s, j}^{(k)} C_{s^{\prime}, j}^{(k)} \delta\left(\omega-\omega_{j}\right) .
$$

This version of the SD represents the $k$ th-order approximant to the full SD resulting from the truncation of the chain Hamiltonian. Below, we will show a series of such approximants for various truncation levels, and we will analyze their convergence for successive truncation levels. Since we refer to reduced-dimensional subspaces, a suitable convolution with a line shape function-here, a Lorentzian-is employed in practice,

$$
J_{s s^{\prime}}^{(k)}(\omega)=\frac{1}{2} \sum_{j=1}^{K} \frac{C_{s, j}^{(k)} C_{s^{\prime}, j}^{(k)} \sigma}{\left(\omega-\omega_{j}\right)^{2}+\sigma^{2}},
$$

where the Lorentzian width parameter $\sigma$ is typically chosen as $\sigma=0.1 \overline{\Delta \omega}$, i.e., as a scaled mean of the average local normal mode frequency spacings $(\overline{\Delta \omega})$.

Alternative formulations of this procedure in a continuous representation can be found in Refs. 36, 37, 39, 42, and 48.

\section{APPLICATION TO STACKED OLIGOTHIOPHENE AGGREGATES}

The $(\mathrm{OT} 4)_{5}$ pentamer is a minimal system permitting a realistic description of spatially extended excitons in an $\mathrm{H}$ type aggregate. We consider an initial system size of 13 electronic states and 195 modes (reduced from a total of 420 normal modes of the pentamer species as further explained below); this full system size is employed in a companion paper $^{47}$ in reference calculations using the ML-MCTDH method. ${ }^{21-24}$ Subsequently, a systematic reduction is carried out using the above effective mode construction. In the present paper, the convergence of the resulting spectral density approximants is analyzed. As illustrated in the companion paper, ${ }^{47}$ convergence at the SD level is found to be directly related to convergence of the dynamical simulations.

In the following, we first report on the details of our electronic structure calculations including the relevant diabatization procedure, followed by the results of the effective mode reduction procedure.

\section{A. Electronic structure calculations and diabatization}

The parameterization of the electronic Hamiltonian of Eq. (2) was based upon supermolecular electronic structure calculations for an $(\mathrm{OT} 4)_{3}$ trimer aggregate representative of a minimal fragment of regioregular rrP3HT. The ground state geometry of the $(\mathrm{OT} 4)_{3}$ species was optimized using DFT calculations with the long-range corrected $\omega \mathrm{B} 97 \mathrm{XD}$ functional ${ }^{51}$ (including empirical
vdW dispersion) and the split valence polarization (SVP) basis set ${ }^{52}$ as implemented in Gaussian09 Rev.D01. ${ }^{53}$ Based on this ground state structure, all relevant parameters for the quantum dynamical simulations were obtained using TDDFT with the same functional, i.e., vertical excitation energies and diabatic couplings of $\widehat{H}_{\text {el }}$ [Eq. (2)] and vibronic couplings of $\widehat{H}_{\text {el-ph }}$ [Eq. (10)]. Due to the pronounced charge transfer characteristics expected for this system, high-level $a b$ initio benchmark calculations at the $\operatorname{ADC}(2)$ level $^{54}$ were performed for the (OT4 $)_{2}$ dimer in order to validate the reliability of the above-mentioned DFT functional. Indeed, the $\omega$ B97XD functional was previously shown to give good results for both excitonic and charge transfer states of P3HT as compared to $\mathrm{ADC}(2) .{ }^{55} \mathrm{We}$ confirmed this observation in our calculations for the (OT4) $)_{2}$ aggregate (see Fig. S1 of the supplementary material).

The diabatic Hamiltonian described above requires obtaining electronic couplings from a diabatization procedure; here, we specifically employed the procedure of Ref. 49. The resulting electronic couplings and on-site energies are summarized in Table I (see Sec. II). These parameters differ to some extent from Ref. 46 where the role of CTX states on charge separation at polythiophenefullerene heterojunctions was addressed. The modified parameterization permits to achieve better agreement with supermolecular calculations and a qualitatively correct representation of the absorption spectrum.

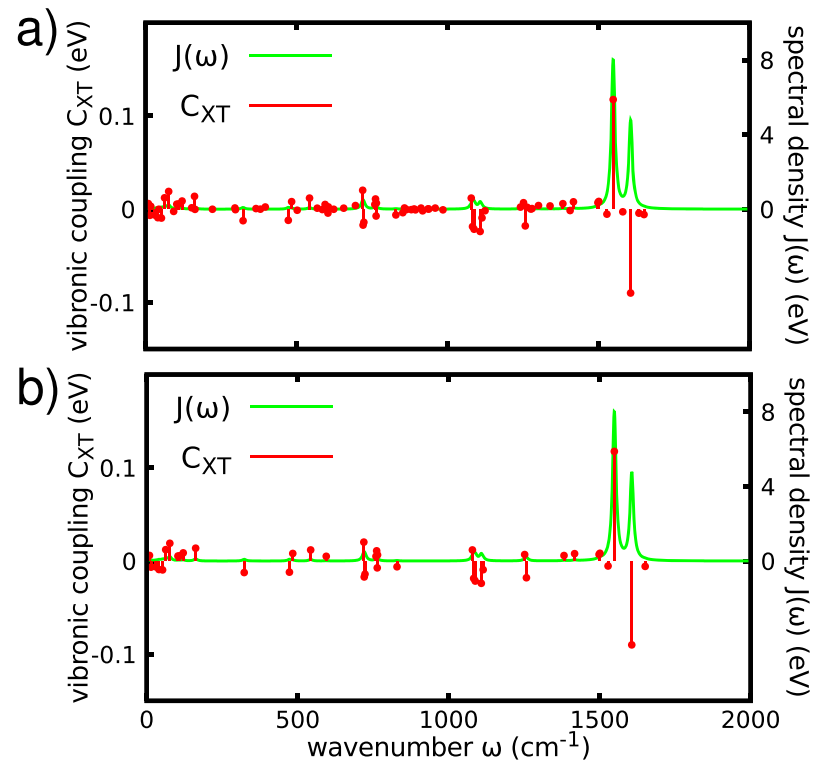

FIG. 3. Calculated vibronic couplings $c_{X T}$ and spectral density $J(\omega)$ for the brigh $S_{2}$ state (of XT type) from excited-state gradients calculated at the TDDFT level for a (OT4) $)_{2}$ dimer. The local modes of a single OT4 fragment have been used for the determination of the vibronic couplings while keeping the other fragment frozen. (a) Full normal mode vibronic couplings and corresponding spectral density obtained by convolution with a Lorentzian $(\Delta=0.1 \overline{\Delta \omega})$. (b) Vibronic couplings above a threshold value of $\left|c_{X T}\right|=0.005 \mathrm{eV}$ and corresponding spectral density. By imposing this threshold, the full number of modes for each OT4 fragment is reduced from 84 to 39 . The reorganization energy changes only marginally by $3 \%$. 
From Table I (see Sec. II), one can see that the difference in on-site energies between the bright XT and CTX states is $0.41 \mathrm{eV}$. The diabatic coupling $\kappa_{1}$ is found to correspond to nearly half of this energy splitting, and $\kappa_{2}$ is non-negligible as well; both will therefore likely give rise to significant mixing between the XT and CTX manifolds. The excitonic coupling $j$ is of the order of $0.1 \mathrm{eV}$ as in our previous analyses ${ }^{48}$ and has a positive sign, which is consistent with an H-type aggregation.

\section{B. Vibronic couplings and spectral densities}

In view of the good performance of the $\omega \mathrm{B} 97 \mathrm{XD}$ DFT functional, vibronic coupling constants were calculated at the TDDFT level. To obtain fragment-specific couplings, the excited-state gradients of the $\left(\mathrm{OT}_{4}\right)_{2}$ dimer at the FC geometry were projected onto the normal modes of one of the fragments. The remaining modes of the other fragment were kept frozen. In the (OT4) dimer, the excited-state characteristics at the FC geometry corresponds approximately to the diabatic states of the Hamiltonian Eqs. (2)-(7) such that the diabatic vibronic couplings are identified with the adiabatic excited-state gradients in the present study. As shown in Fig. S5 of the supplementary material, the vibronic couplings computed for the OT4 model system are representative of P3HT oligomers.

Figure 3(a) shows the $\boldsymbol{c}_{\mathrm{XT}}$ vibronic coupling constants for a single OT4 fragment, obtained from the excited-state gradient of the bright $S_{2}$ state of the (OT4) $)_{2}$ dimer which exhibits XT characteristics. In the same panel, the associated spectral density obtained by convolution with a Lorentzian according to Eq. (35) is shown. Like-

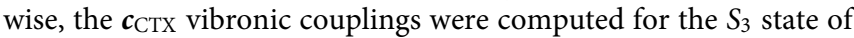
the $(\mathrm{OT} 4)_{2}$ dimer which exhibits charge-transfer excitonic characteristics. It turns out that the relevant vibronic couplings show a similar frequency dependence but different amplitudes for the XT vs
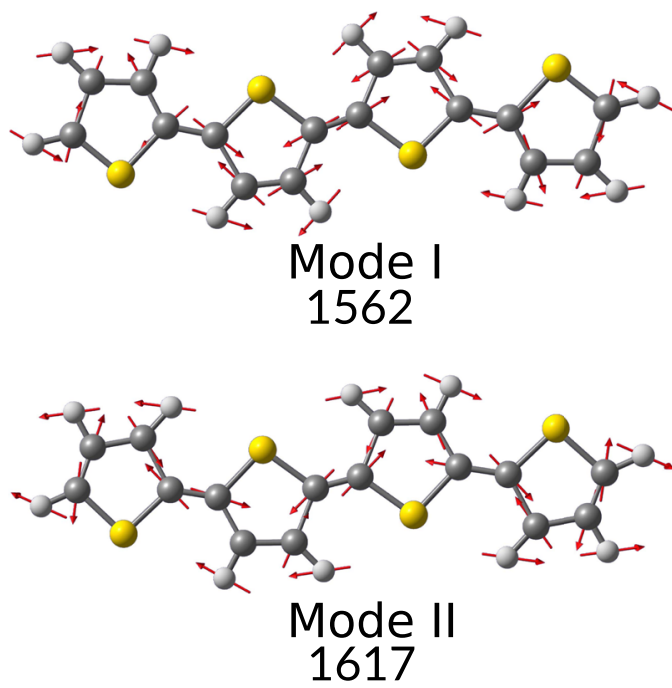

FIG. 4. Visualization of the two most strongly coupled normal modes. Mode I corresponds to the 76th normal mode of one OT4 fragment, while mode II corresponds to the 78th normal mode. Frequencies are indicated in $\mathrm{cm}^{-1}$.
CTX states (see Figs. S6-S8 of the supplementary material). Notably, the vibronic couplings take larger values for the CTX states, showing that reorganization effects are more pronounced in the CTX states.

The monomer XT spectral density shown in Fig. 3(a) exhibits vibronic couplings in the full normal-mode space spanned by 84 modes. Among these, two modes around $\sim 1550-1600 \mathrm{~cm}^{-1}$ are dominant which are collective modes involving symmetric (mode I) and antisymmetric (mode II) stretching of the aromatic $\mathrm{C}=\mathrm{C}$ bonds, combined with $\mathrm{C}-\mathrm{C}$ displacements of the conjugated backbone ${ }^{56}$ (see Fig. 4). These two modes exhibit large vibronic coupling values since they entail deformations within the $\pi$ electronic structure and therefore influence the optical properties of the system. Furthermore, a large number of small vibronic couplings are present, covering the full frequency range. The influence of these vibronic couplings on the reorganization energy $\lambda_{\mathrm{XT}}=\sum_{n} c_{\mathrm{XT}, n}^{2} /\left(2 \omega_{n}\right)$ is small such that we introduced a threshold value of $c_{\mathrm{XT}, n}=0.005 \mathrm{eV}$, below which the couplings were neglected. Figure $3(\mathrm{~b})$ shows the reduced set of vibronic couplings-now for 39 modes-after imposing this threshold value. The difference in reorganization energy $\lambda_{\mathrm{XT}}$ between the full normal mode representation and the reduced set of
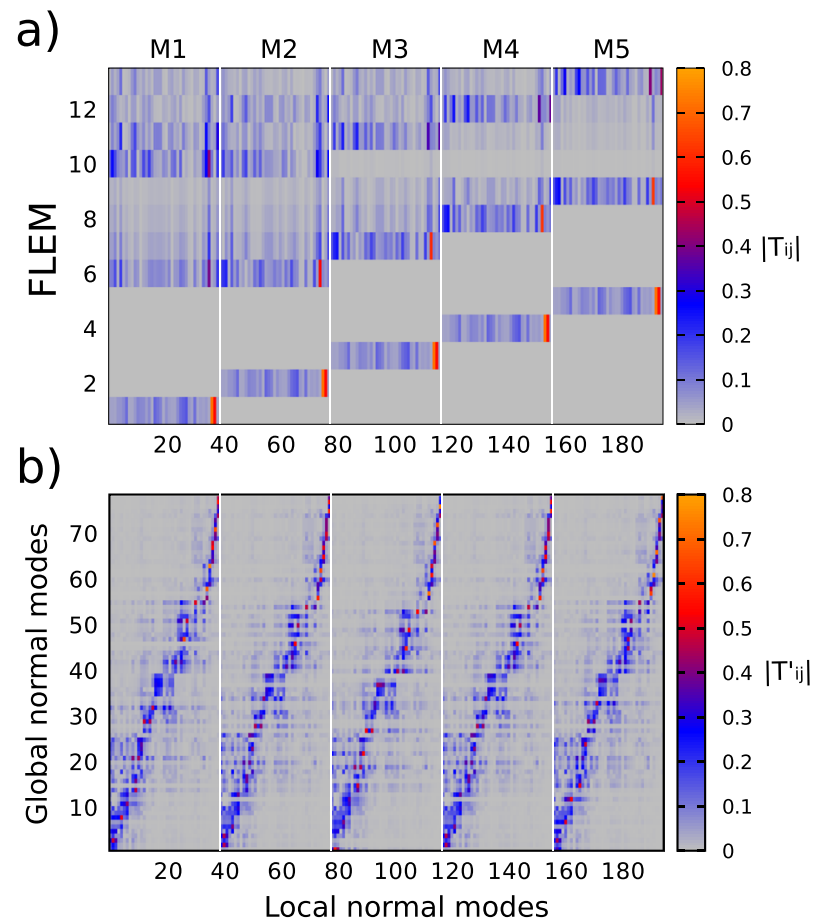

FIG. 5. Graphical representation of effective modes in the basis of the original local NMs. Within each local NM subspace, modes are shown in order of ascending frequency. (a) First layer effective mode (FLEM) space of dimension $N_{s}=13$ in the band-diagonal representation. The absolute values of the transformation matrix elements $T_{i j}, i=1, \ldots, N_{s}, j=1, \ldots, N_{\text {ph }}$, according to Eq. (23) are shown. (b) Truncated effective mode space for $k=6$ (truncation at the sixth order), leading to $K=k N_{s}=78$ modes; the rediagonalized representation is shown, i.e., global NMs of the truncated effective Hamiltonian. The graphical representation shows absolute values of the transformation matrix elements $T_{i j}^{\prime}, i=1, \ldots, k N_{s}$, $j=1, \ldots, N_{\text {ph }}$, where the transformation $T^{\prime}$ is a concatenation of Eq. (23) and the additional transformations of Eqs. (29) and (30). 
normal modes is $3 \%$. The same set of modes is retained for the CTX states.

As detailed in the companion paper, ${ }^{47}$ the above approximation permits us to reduce the overall system size such that ML-MCTDH reference calculations can be conducted for the (OT4) $)_{5}$ pentamer system for a model involving 13 electronic states and 195 modes. This system size is therefore taken as a reference for the present assessment of the effective-mode reduction as well.

\section{Effective mode transformation and spectral density approximants}

The effective mode transformation as described in Sec. III is now illustrated for the (OT4) 5 pentamer species. To start with, Fig. 5 shows the FLEM subspace in the basis of local NMs [panel (a)]. In a complementary fashion, the full set of effective modes is shown in Fig. S10 of the supplementary material, illustrating that the first few layers feature high-frequency and low-frequency modes in alternation, as in related systems that we investigated. ${ }^{32-34,50}$ Following the rediagonalization procedure described in Sec. III, the final set of effective modes is obtained, which correspond to the normal modes of the truncated effective-mode space. Figure 5(b) shows this final set of effective modes, resulting from a truncation at the order $k=6$ such that the band-diagonal representation of Eq. (29) spans $K=k N_{s}=78$ modes. The rediagonalized representation provides an equivalent representation within the truncated space and is employed for the SD reconstruction and quantum propagation.

As discussed above, five of the 13 FLEM modes are of local characteristics by construction and subsume the XT vibronic couplings, whereas the remaining eight effective modes relate to the CTX vibronic couplings and require initial orthogonalization. As a result, these latter modes are delocalized across the fragments [see Fig. 5(a)]. In the complementary representation of
Fig. 5(b) -in the normal mode representation of the truncated effective-mode space-all modes are delocalized across the fragments and therefore appear as collective vibrations across the aggregate. As discussed in Sec. III C, the normal-mode representation in the truncated effective-mode space naturally yields a global NM picture. Each of these global NMs is seen to cover a narrow band of frequencies.

Convergence of the effective mode chain is first analyzed for the $(\mathrm{OT} 4)_{2}$ dimer species. The $k$ th order SD matrix of Eq. (35) is constructed for four electronic states (two XT states and two CTX states), and the convergence properties as a function of $k$ are illustrated in Fig. 6. For reference, the full SD in the fragment normal-mode representation, with 39 modes, is shown in black. The SDs resulting from a truncation at the orders $k=1, \ldots, 4$ with up to 16 effective modes (depicted in gray) differ significantly from the frequency dependence and shape of the normal-mode SD. By including two more layers of effective modes (i.e., $k=6$, shown in red), the spectral density becomes bimodal, but the intensity ratio is not yet correct. Finally, for eight layers (i.e., $k=8$, shown in green), convergence is very good, and the SD exhibits the correct intensity ratio between the two prominent high-frequency peaks.

Since convergence at the $k=8$ order was found to be good in the case of the (OT4) $)_{2}$ system, we adopt the same level of treatment for our target system, the (OT4) 5 pentamer. As shown in Fig. 7, the SD is again in excellent agreement with the reference normal-mode SD at this order, both for the diagonal and off diagonal components. At this level, the number of modes has been reduced from 195 to 104 . The reduction to the $k=6$ level (78 modes), which is qualitatively correct even though amplitudes tend to deviate visibly, is shown in Fig. S9 of the supplementary material. The SD matrix of Fig. 7 illustrates that correlated fluctuations are a prominent feature of the system; in fact, the off diagonal SD contributions are of the same magnitude as the diagonal a)

b)
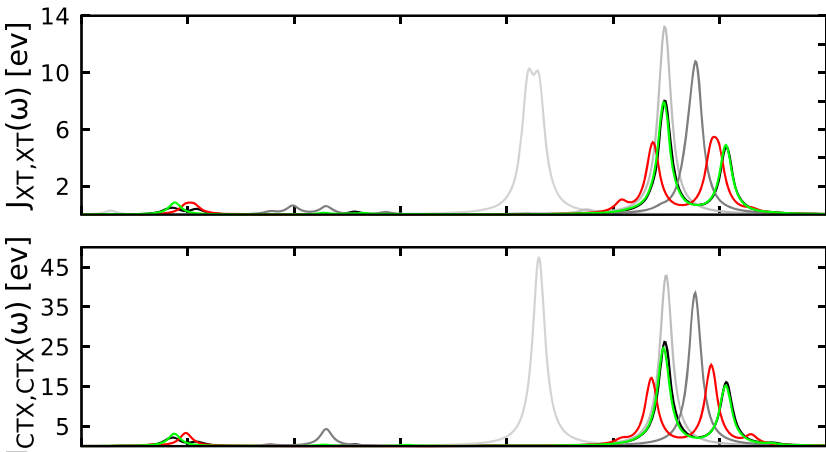

C)

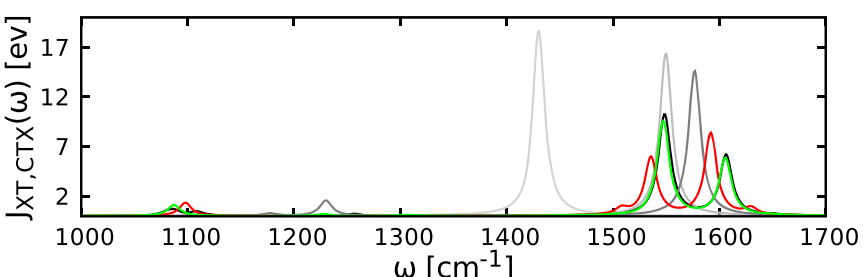

FIG. 6. For the $(\mathrm{OT} 4)_{2}$ dimer, the convergence behavior of the $J_{\mathrm{XT}}^{(k)}$ [panel (a)], $J_{\text {CTX.СTX }}^{(k)}$ [panel (b)], and $J_{\text {XT,CTX }}^{(k)}$ [panel (c)] component spectral densities is shown for different truncation orders $k$ of the effective-mode hierarchy, as compared with the corresponding original spectral densities in the local NM representation (shown in black). For the dimer species, $N_{s}=4$ such that the number of effective modes is given as $K=4 k$ The effective mode spectral densities comprising up to 16 effective modes are drawn in gray, while those comprising 24 $(k=6)$ and $32(k=8)$ effective modes are depicted in red and green, respectively. Note the different scales for the SD components; specifically, the diagonal CTX SD takes a large value, reflecting large reorganization effects in the CTX state. All spectral densities have been constructed with a Lorentzian broadening of $\Delta=0.1 \overline{\Delta \omega}$ 

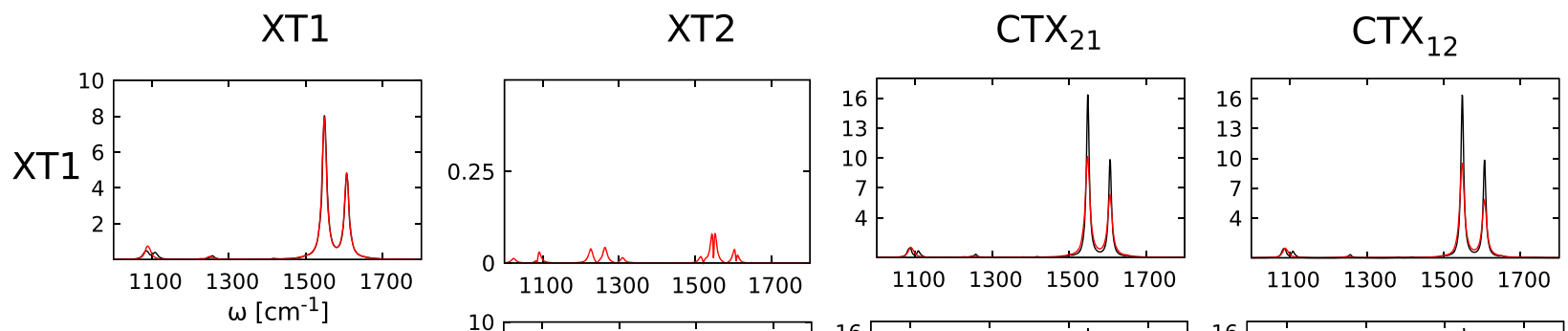

$\mathrm{XT2}$
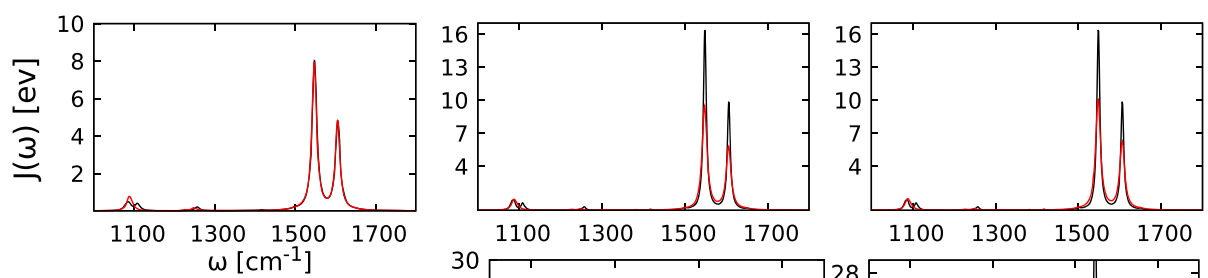

CTX

21

CTX12

normal modes
104 effective modes
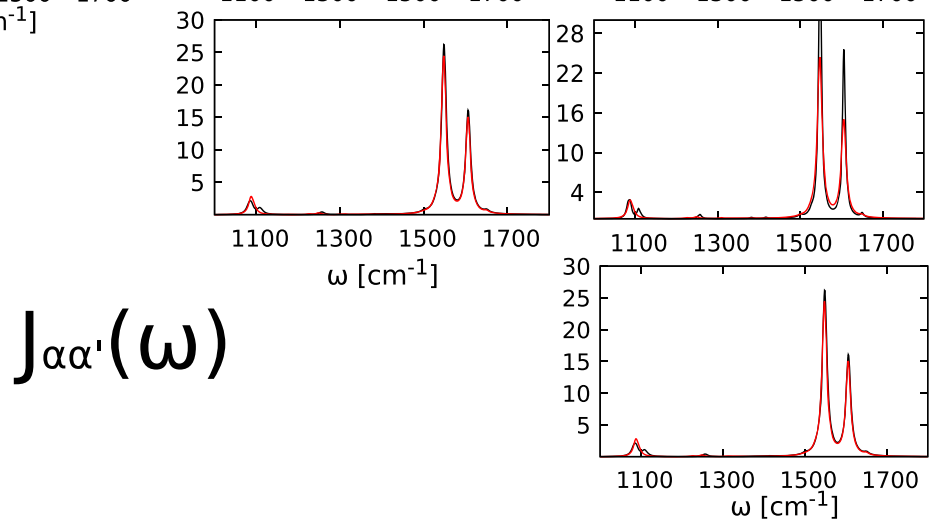

FIG. 7. For the (OT4) 5 pentamer, selected elements (absolute values) of the spectral density matrix $J^{(k=8)}$, i.e., for $K=104$ effective modes, are shown (red traces), as compared with the reference spectral density in the original local NM representation (black traces). It is seen that all diagonal and off diagonal spectral density components exhibit a similar frequency dependence, mainly characterized by the bimodal feature that is already prominent in the monomer spectral density. Note that XT-XT cross correlations vanish by construction (and are almost zero at the $k=8$ level of treatment). The convergence at the $k=8$ order is very good and of similar quality as observed in the dimer case of the preceding figure.

contributions. Note, though, that the XT-XT cross correlations vanish by construction due to the local nature of the electronic operators and the site-local modes.

From a dynamical perspective, we anticipate that vibronic XT-CTX correlations are going to drive a correlated multistate wavepacket dynamics. As described in the companion paper ${ }^{47}$ on quantum dynamical simulations using the present vibronic Hamiltonian, a correlated, coherent transfer dynamics between the XT and CTX manifolds is indeed observed. A striking feature is the regular modulation of all electronic state populations with a frequency of $\sim 22 \mathrm{fs}$ which corresponds to the dominant SD modes. Hence, correlated vibronic effects are directly observable in the multistate dynamics.

As also detailed in the companion paper, ${ }^{47}$ the convergence of the spectral densities is used as a guideline for the number of effective modes that should be included in the quantum dynamical treatment. Indeed, ML-MCTDH simulations at the $k=6$ order (13 electronic states and 78 modes) can be shown to reproduce the full dynamics (13 states and 195 modes) quite accurately, and the $k=8$ order brings further improvement.

\section{CONCLUSION}

This paper demonstrates the construction and application of reduced effective-mode schemes for the description of vibronic coupling in donor-acceptor aggregates where fragment vibrations couple both to local excitations (Frenkel states) and chargetransfer excitonic states. We demonstrated the method for a homoaggregate of stacked oligothiophene species, representative of similar instances of mixed Frenkel and charge-transfer excitons, e.g., in pentacene ${ }^{57}$ and perylenes. ${ }^{58}$ Furthermore, the generalization to hetero-aggregates is straightforward, i.e., different on-site energies and monomer-specific spectral densities are easily accommodated within the effective-mode construction scheme. Hence, the present procedure could be straightforwardly transposed to a variant of the $(\mathrm{OT} 4)_{n}$ Hamiltonian containing static disorder effects or else to related cases like charge transfer excitons in DNA. ${ }^{3,4}$

Following our earlier treatments, notably involving conical intersection topologies in polyatomic systems, ${ }^{27,42}$ the effectivemode approach is employed in the framework of a linear vibronic 
coupling model. ${ }^{25}$ In the absence of large amplitude motions, this approximation is suitable and offers the advantage of connecting to model Hamiltonians and system-bath theory approaches where spectral densities appear as the key quantities. Furthermore, the model in its present form could be combined with the explicit inclusion of selected low-frequency anharmonic modes, e.g., intermolecular modes and torsional degrees of freedom.

In practice, the construction of the LVC model Hamiltonian for the (OT4) $)_{n}$ systems under study involves the determination of electronic energies and diabatic couplings, which were here calculated by a suitable quasidiabatization scheme. ${ }^{49}$ Intrastate vibronic couplings were determined by projecting the excitedstate gradients onto the normal-mode space of individual fragments.

As explained above, the effective-mode approach is based upon the truncation of the LVC Hamiltonian when transformed to a chain representation. In the present procedure, we perform an additional transformation which generates the normal modes of the truncated chain Hamiltonian, whose coupling to the electronic subspace is again of LVC type. These modes correspond to global, delocalized normal modes of the entire system. This procedure permits the reconstruction of a series of spectral density approximants without having recourse to the continued-fraction representation of the SDs that results from the chain representation of the Hamiltonian. ${ }^{36-40,42}$ Furthermore, the use of a reduced-dimensional Hamiltonian in the LVC form is straightforward in the context of quantum dynamical propagation, where the bilinear coordinate and momentum couplings of the chain representation prove numerically demanding.

In the present discussion, the level of truncation of the effective mode hierarchy was determined by the reconstruction of the spectral density. This generally requires higher truncation orders than reproducing, e.g., the first few moments of linear absorption spectra, which is guaranteed by the FLEM space. ${ }^{27-30}$ In the present treatment, the FLEMs need to be complemented by additional layers comprising lower-frequency modes to reproduce the full SD in a qualitatively correct fashion. This coincides with the observationsimilar to our previous studies of simpler systems ${ }^{32-34}$ - that lowfrequency modes play an essential role in the dynamics, even though high-frequency modes dominate the vibronic coupling. In the present case, this observation underscores that a dynamical approximation which entirely relies on the most prominent highfrequency modes of the original spectral density tends to be inaccurate beyond the shortest time scale, as further detailed in our companion paper.

Effective mode schemes, which are closely related to timedependent density matrix renormalization group (DMRG) approaches, ${ }^{59}$ provide a flexible strategy to describe vibronic coupling in molecular aggregates. The case of correlated fluctuations of electronic subsystem variables induced by the vibrational modes is of particular interest in this context, and the effective-mode treatment is naturally suited to capture these correlations. Given that vibronic interactions are a ubiquitous phenomenon in molecular aggregates where dense manifolds of electronic states interact and electronic delocalization prevails, correlated vibronic effects are expected to play an important role and contribute to the coherent, collective nature of the dynamics.

\section{SUPPLEMENTARY MATERIAL}

See supplementary material for various additional electronic structure results and details of the effective-mode analysis.

\section{ACKNOWLEDGMENTS}

We thank Dr. Hiroyuki Tamura (Tokyo University) for discussions regarding the parameterization of the aggregate model. Funding by the DFG (Grant No. BU-1032-2) is gratefully acknowledged.

\section{REFERENCES}

${ }^{1}$ V. May and O. Kühn, Charge and Energy Transfer Dynamics in Molecular Systems, 3rd ed. (VCH-Wiley, 2011).

${ }^{2}$ Ultrafast Dynamics at the Nanoscale: Biomolecules and Supramolecular Assemblies, edited by I. Burghardt and S. Haacke (Pan Stanford Publishers, Singapore, 2017).

${ }^{3}$ L. Serrano-Andrés, M. Mechán, and A. C. Borin, Chem. Eur. J. 12, 6559 (2006).

${ }^{4}$ J. J. Nogueira, F. Plasser, and L. González, Chem. Sci. 8, 5682 (2017).

${ }^{5}$ P. Huo and D. F. Coker, J. Chem. Phys. 133, 184108 (2010).

${ }^{6}$ J. Schulze, M. F. Shibl, M. J. Al-Marri, and O. Kühn, J. Chem. Phys. 144, 185101 (2016).

${ }^{7}$ A. Köhler and H. Bässler, Electronic Processes in Organic Semiconductors (VCHWiley, 2015).

${ }^{8}$ W. Barford, Electronic and Optical Properties of Conjugated Polymers, 2nd ed. (Clarendon, Oxford, 2013).

${ }^{9}$ G. Lanzani, The Photophysics Behind Photovoltaics and Photonics (Wiley VCH, Singapore, 2012).

${ }^{10}$ N. S. Sariciftci, L. Smilowitz, A. J. Heeger, and F. Wudl, Science 258, 1474 (1992).

${ }^{11}$ C. Deibel, T. Strobel, and V. Dyakonov, Adv. Mater. 22, 4097 (2010).

${ }^{12}$ S. M. Ryno, M. K. Ravva, X. Chen, H. Li, and J. Brédas, Adv. Energy Mater. 7, 1601370 (2017).

${ }^{13}$ K. Tremel and S. Ludwigs, Adv. Polym. Sci. 265, 39 (2014).

${ }^{14}$ F. C. Spano, Annu. Rev. Phys. Chem. 57, 217 (2006).

${ }^{15}$ A. T. Kleinschmidt, S. E. Root, and D. J. Lipomi, J. Mater. Chem. A 5, 11396 (2017).

${ }^{16}$ A. De Sio and C. Lienau, Phys. Chem. Chem. Phys. 19, 18813 (2017).

${ }^{17}$ M. Polkehn, P. Eisenbrandt, H. Tamura, and I. Burghardt, Int. J. Quantum Chem. 118, e25502 (2018).

${ }^{18}$ T. Unger, S. Wedler, F. Kahle, U. Scherf, H. Bässler, and A. Köhler, J. Phys. Chem. C 121, 22739 (2017).

${ }^{19}$ H.-D. Meyer, U. Manthe, and L. S. Cederbaum, Chem. Phys. Lett. 165, 73 (1990).

${ }^{20}$ M. H. Beck, A. Jäckle, G. A. Worth, and H.-D. Meyer, Phys. Rep. 324, 1 (2000).

${ }^{21}$ G. A. Worth, H.-D. Meyer, H. Köppel, L. S. Cederbaum, and I. Burghardt, Int. Rev. Phys. Chem. 27, 569 (2009).

${ }^{22}$ G. A. Worth, M. H. Beck, A. Jäckle, and H.-D. Meyer, The MCTDH package, version 8.5, 2014, see http://www.pci.uni-heidelberg.de/tc/usr/mctdh.

${ }^{23}$ H. Wang, J. Chem. Phys. 119, 1289 (2003).

${ }^{24}$ O. Vendrell and H.-D. Meyer, J. Chem. Phys. 134, 044135 (2011).

${ }^{25}$ H. Köppel, W. Domcke, and L. S. Cederbaum, Adv. Chem. Phys. 57, 59 (1984).

${ }^{26}$ U. Weiss, Quantum Dissipative Systems, 3rd ed. (World Scientific Singapore, 2008).

${ }^{27}$ L. S. Cederbaum, E. Gindensperger, and I. Burghardt, Phys. Rev. Lett. 94, 113003 (2005).

${ }^{28}$ E. Gindensperger, I. Burghardt, and L. S. Cederbaum, J. Chem. Phys. 124, 144103 (2006).

${ }^{29}$ E. Gindensperger, I. Burghardt, and L. S. Cederbaum, J. Chem. Phys. 124, 144104 (2006).

${ }^{30}$ I. Burghardt, E. Gindensperger, and L. S. Cederbaum, Mol. Phys. 104, 1081 (2006). 
${ }^{31}$ H. Mori, Prog. Theor. Phys. 34, 399 (1965).

${ }^{32}$ H. Tamura, E. R. Bittner, and I. Burghardt, J. Chem. Phys. 126, 021103 (2007).

${ }^{33}$ H. Tamura, E. R. Bittner, and I. Burghardt, J. Chem. Phys. 127, 034706 (2007).

${ }^{34}$ H. Tamura, J. G. S. Ramon, E. R. Bittner, and I. Burghardt, Phys. Rev. Lett. 100, 107402 (2008)

${ }^{35}$ E. Gindensperger, H. Köppel, and L. S. Cederbaum, J. Chem. Phys. 126, 034106 (2007).

${ }^{36}$ K. H. Hughes, C. D. Christ, and I. Burghardt, J. Chem. Phys. 131, 024109 (2009).

${ }^{37}$ K. H. Hughes, C. D. Christ, and I. Burghardt, J. Chem. Phys. 131, 124108 (2009).

${ }^{38}$ R. Martinazzo, K. H. Hughes, F. Martelli, and I. Burghardt, Chem. Phys. 377, 21 (2010).

${ }^{39}$ R. Martinazzo, B. Vacchini, K. H. Hughes, and I. Burghardt, J. Chem. Phys. 134, 011101 (2011)

${ }^{40}$ R. Martinazzo, K. H. Hughes, and I. Burghardt, Phys. Rev. E 84, 030102(R) (2011).

${ }^{41}$ R. Martinazzo, K. H. Hughes, and I. Burghardt, "Hierarchical effective-mode approach for extended molecular systems," in Advances in the Theory of Quantum Systems in Chemistry and Physics, edited by P. E. Hoggan, E. J. Brändas, J. Maruani, P. Piecuch, and G. Delgado-Barrio (Springer, 2012), pp. 269-284.

${ }^{42}$ I. Burghardt, K. H. Hughes R. Martinazzo, H. Tamura, E. Gindensperger, H. Köppel, and L. S. Cederbaum, "Conical intersections coupled to an environment," in Conical Intersections, edited by W. Domcke, D. Yarkony, and H. Köppel (World Scientific, 2011), pp. 301-346.

${ }^{43}$ N. J. Hestand and F. C. Spano, J. Chem. Phys. 143, 244707 (2015).

${ }^{44}$ O. G. Reid, R. D. Pensack, Y. Song, G. D. Scholes, and G. Rumbles, Chem. Mater. 26, 561 (2013).

${ }^{45}$ T. J. Magnanelli and A. E. Bragg, J. Phys. Chem. Lett. 6, 438 (2015).

${ }^{46} \mathrm{M}$. Polkehn, H. Tamura, and I. Burghardt, J. Phys. B: At. Mol. Opt. Phys. 51, 014003 (2018)

${ }^{47}$ W. Popp, M. Polkehn, R. Binder, and I. Burghardt, J. Phys. Chem. Lett. 10, 3326 (2019).
${ }^{48}$ M. Huix-Rotllant, H. Tamura, and I. Burghardt, J. Phys. Chem. Lett. 6, 1702 (2015).

${ }^{49}$ L. Blancafort and A. A. Voityuk, J. Chem. Phys. 140, 095102 (2014).

${ }^{50}$ H. Tamura, J. Ramon, E. R. Bittner, and I. Burghardt, J. Phys. Chem. B 112, 495 (2008).

${ }^{51}$ J.-D. Chain and M. Head-Gordon, Phys. Chem. Chem. Phys. 10, 6615 (2008).

${ }^{52}$ A. Schäfer, H. Horn, and R. Ahlrichs, J. Chem. Phys. 97, 2571 (1992).

${ }^{53}$ M. J. Frisch, G. W. Trucks, H. B. Schlegel, G. E. Scuseria, M. A. Robb, J. R. Cheeseman, G. Scalmani, V. Barone, B. Mennucci, G. A. Petersson, H. Nakatsuji, M. Caricato, X. Li, H. P. Hratchian, A. F. Izmaylov, J. Bloino, G. Zheng, J. L. Sonnenberg, M. Hada, M. Ehara, K. Toyota, R. Fukuda, J. Hasegawa, M. Ishida, T. Nakajima, Y. Honda, O. Kitao, H. Nakai, T. Vreven, Montgomery, J. A. Peralta, J. E. Peralta, F. Ogliaro, M. Bearpark, J. J. Heyd, E. Brothers, K. N. Kudin, V. N. Staroverov, R. Kobayashi, J. Normand, K. Raghavachari, A. Rendell, J. C. Burant, S. S. Iyengar, J. Tomasi, M. Cossi, N. Rega, J. M. Millam, M. Klene, J. E. Knox, J. B. Cross, V. Bakken, C. Adamo, J. Jaramillo, R. Gomperts, R. E. Stratmann, O. Yazyev, A. J. Austin, R. Cammi, C. Pomelli, J. W. Ochterski, R. L. Martin, K. Morokuma, V. G. Zakrzewski, G. A. Voth, P. Salvador, J. J. Dannenberg, S. Dapprich, A. D. Daniels, Ö. Farkas, J. B. Foresman, J. V. Ortiz, J. Cioslowski, and D. J. Fox, Gaussian 09, Revision E.01, Gaussian, Inc., Wallingford, CT, 2009.

${ }^{54}$ A. B. Trofimov and J. Schirmer, J. Phys. B: At., Mol. Opt. Phys. 28, 2299 (1995).

${ }^{55}$ H. Li, R. Nieman, A. J. A. A. H. Lischka, and S. Tretiak, J. Chem. Theory Comput. 10, 3280 (2014).

${ }^{56}$ J. Casado, S. Hotta, V. Hernández, and J. T. López Navarette, J. Phys. Chem. A 103, 816 (1999).

${ }^{57}$ S. Sharifzadeh, P. Darancet, L. Kronik, and J. B. Neaton, J. Phys. Chem. Lett. 4, 2197 (2013).

${ }^{58}$ L. Gisslén and R. Scholz, Phys. Rev. B 80, 115309 (2009).

${ }^{59}$ S. R. White and A. E. Feiguin, Phys. Rev. Lett. 93, 076401 (2004). 